\title{
ConCerv: a prospective trial of conservative surgery for low-risk early stage cervical cancer, by Schmeler et al
}

Gynecology Service, Department of Surgery, Memorial Sloan Kettering Cancer Center, New York, New York, USA

Correspondence to Dr Nadeem R Abu-Rustum, Department of Surgery, Memorial Sloan Kettering Cancer Center, New York NY10065, USA; abu-rusn@ mskcc.org

Received 9 August 2021 Accepted 11 August 2021 Published Online First 24 September 2021

\section{Linked}

- http://dx.doi.org/10.1136/ ijgc-2021-002921

\section{Check for updates}

(C) IGCS and ESGO 2021. № commercial re-use. See rights and permissions. Published by BMJ.

To cite: Abu-Rustum NR Int J Gynecol Cancer

2021;31:1326-1327.

Nadeem R Abu-Rustum (D)

I would like to congratulate $\mathrm{Dr}$ Schmeler and colleagues on completing an important, welldesigned, and well-monitored study evaluating the feasibility and oncologic outcomes of conization alone or simple hysterectomy (conservative surgery for fertility preservation) in stage IA2-IB1 cervical cancers of squamous histology and adenocarcinomas. The ConCerv study ${ }^{1}$ highlights many aspects of the expert management of early-stage cervical cancer and sets standards for selection criteria that ensure patient safety when less radical surgery is used in select stage IA2-IB1 cases. The meticulous selection criteria, centralized expert pathology review, and diligent review and modification of the inclusion criteria by the data safety monitoring committee are major strengths that should reassure physicians and patients when opting for this conservative approach.

Cervical conization has emerged as one of the most powerful tools in the triage of apparent earlystage cervical cancer. The importance of cervical conization with endocervical curettage above the cone apex cannot be overemphasized in this selection process. Conization with expert gynecologic pathology review allows for the confirmation of an International Federation of Gynecology and Obstetrics (FIG0) stage IA2-IB1 carcinoma, confirms a favorable histology and grade (anygrade squamous cell carcinoma or grade $1 / 2$ usual-type adenocarcinoma), and confirms the absence of Iymphovascular invasion. Conization also ensures the selection of cases with depth of invasion $\leq 10 \mathrm{~mm}$, with negative margins and endocervical curettage for malignancy or highgrade dysplasia. The ConCerv study defines a negative cone margin as no invasive cancer within $1.0 \mathrm{~mm}$ of both the endocervical and ectocervical margins and no adenocarcinoma in situ, cervical intraepithelial neoplasia 2 or 3 at the inked or cauterized margin. This is an important contribution, as gynecologic oncologists and pathologists continue to discuss the definition of a negative margin on conization. The importance of physical examination and imaging to ensure tumor size $<2$ $\mathrm{cm}$ and exclude obvious metastatic disease is also highlighted by the inclusion criteria.

The ConCerv study also emphasizes the power of a negative conization margin to predict residual carcinoma in follow-up hysterectomy specimens (only one (2.5\%) of 40 specimens - an adenocarcinoma); for carcinomas of squamous histology, a negative conization margin was fully reliable in predicting no residual disease on hysterectomy. Furthermore, ConCerv provides strong evidence for the safety of eliminating parametrectomy and upper vaginectomy in these very well-selected cases; however, the study underscores the importance of continuing the practice of pelvic lymphadenectomy or sentinel lymph node mapping even in the most favorable selected stage IA2-IB1 lesions. Five patients (5\% (all with squamous lesions) had positive pelvic nodes, which profoundly changes stage and management. Taken together, we can conclude that when applying the strict ConCerv selection criteria, parametrectomy and upper vaginectomy could be safely eliminated.

Ninety-six percent of cases in the ConCerv trial were performed via a minimally invasive approach (robotic-assisted or laparoscopy), and although not an objective of the study, these data confirm previous reports that conization and removal of the cervical lesion may play a role in the safety of minimally invasive surgery in early-stage cervical cancer. $^{23}$

The ConCerv trial also gives us insight into the management of select stage IA2-IB1 cancers detected after inadvertent simple hysterectomy with negative hysterectomy margins and inclusion criteria as defined above. Even though the data safety monitoring committee correctly recommended stopping the inclusion of these cases after two $(12.5 \%)$ of 16 patients recurred, it is not clear whether these cases were truly due to limiting surgical radicality (one recurrence to both the pelvis and lung and one to the inguinal region); therefore, these data also provide good insight into the management of very select stage IA2-IB1 cases detected after inadvertent simple hysterectomy 
who meet the strict ConCerv criteria and have negative bilateral pelvic nodes.

Finally, the importance of data and safety monitoring committee review in prospective clinical trials to refine inclusion criteria and maintain patient safety cannot be overemphasized. The authors should be commended on refining their inclusion criteria as the study progressed by limiting the depth of invasion to $\leq 10 \mathrm{~mm}$ and restricting cases detected on inadvertent hysterectomy. The data from this international collaboration provide reassuring management options for very select cases of stage IA2-IB1 cervical cancer. The ConCerv criteria will be used increasingly over time and will contribute to changing the standard of care in this disease.

Contributors NRA-R is the sole contributor.

Funding The author has not declared a specific grant for this research from any funding agency in the public, commercial or not-for-profit sectors.
Competing interests NRA-R reports grants (paid to the institution) from Stryker/ Novadaq and GRAIL, outside the submitted work.

Patient consent for publication Not required.

Provenance and peer review Commissioned; internally peer reviewed.

\section{ORCID iD}

Nadeem R Abu-Rustum http://orcid.org/0000-0001-9689-1298

\section{REFERENCES}

1 Schmeler KM, Pareja R, Lopez Blanco A, et al. ConCerv: a prospective trial of conservative surgery for low-risk early-stage cervical cancer. Int J Gynecol Cancer 2021;31:1317-25.

2 Chiva L, Zanagnolo V, Querleu D, et al. SUCCOR study: an international European cohort observational study comparing minimally invasive surgery versus open abdominal radical hysterectomy in patients with stage IB1 cervical cancer. Int J Gynecol Cancer 2020;30:1269-77.

3 Chiva L, Chacon E. Is conization a protective surgical maneuver in early cervical cancer? Ann Surg Oncol 2021;28:3463-4. 